\title{
Adrenal Myelolipoma with Adrenocortical Adenoma Presenting with Hypertension Only
}

\author{
Fan Yang*1, Chenyu Sun*2, Shiyu Pang1, Juanjuan Cai3 , Hao Lin ${ }^{1}$ and Wanlong Tan
}

\begin{abstract}
Here, we present a case of a 25-year Chinese female who was diagnosed with non-functional adrenocortical adenoma containing myelolipoma with hypertension as the only symptom. Serum levels of cortisol, aldosterone, angiotensin $\mathrm{I} / \mathrm{Il}$ and renin activity were normal. Myelolipoma is a benign, non-functioning retroperitoneal tumour occurring predominantly in the adrenal gland and relatively uncommon. With the advancement of radiological studies, the incidental detection of myelolipoma has been noted. However, the coexistence of adrenal myelolipoma and adrenal adenoma still remains extremely rare. Though usually benign, the later may present with endocrine dysfunction, such as Cushing's syndrome, and requires proper management. Surgical resection is reserved for symptomatic tumours or large myelolipoma $(>7 \mathrm{~cm}$ in size). The final diagnosis mainly relies on pathological examination. The left adrenal mass was completely removed via retroperitoneal laparoscopic approach. Postoperative recovery was uneventful and her blood pressure (BP) readings were normal. At 15 months follow-up, the patient was normotensive and there was no recurrence of tumour.
\end{abstract}

Key Words: Adrenal gland, Myelolipoma, Adrenocortical adenoma, Surgical resection.

\section{INTRODUCTION}

Adrenal myelolipoma is a benign, non-functioning tumour occurring predominantly in the adrenal gland and is relatively uncommon.1,2 Most myelolipomas were discovered at autopsy in the past, with an incidence rate of $0.2 \% .{ }^{1}$ With the advancement of radiological studies, the incidental detection of myelolipoma has been reported more often by using ultrasonography, magnetic resonance imaging (MRI), and computed tomography (CT). ${ }^{3}$ Despite increasing detection of adrenal myelolipoma, the coexistence of adrenal myelolipoma and adrenal adenoma still remains extremely rare. 4

Here, we present a case of a 25 -year Chinese female who had non-functional adrenocortical adenoma containing myelolipoma with hypertension as the only symptom. She underwent successful surgical resection and is symptom-free at last follow-up.

\section{CASE REPORT}

A 25-year Chinese female with a two-year history of hypertension was referred to the hospital. She underwent open surgery of a mediastinal lymphangioma two

1 Department of Urology, Nanfang Hospital, Southern Medical University, Guangzhou, Guangdong, 510515-China

2 The First Affiliated Hospital of Anhui Medical University, Hefei, Anhui, 230022-China

3 Department of Pathology, Zhejiang Provincial People's Hospital, Hangzhou, Zhejiang, 310000-China

Correspondence: Dr. Wanlong Tan, Department of Urology, Nanfang Hospital, Southern Medical University, Guangzhou, Guangdong, 510515-China

E-mail: tanwlurologist@126.com

Received: August 29, 2018; Accepted: December 19, 2018

*These authors contributed equally. years ago in a local hospital. Her blood pressure (BP) was elevated, with the highest record of $158 / 112 \mathrm{mmHg}$. Due to lack of hypertension-related symptoms, she did not take any medication during the 20 months after surgery; until a routine follow-up CT scan revealed a mass in the left upper retroperitoneal region.

On physical examination at admission, she was alert with no acute distress. Vital signs were normal except for elevated BP of 149/104 mmHg. A $20 \mathrm{~cm}$ long surgical incision scar was visible on the right axilla. Previous history included an abortion two years ago. No acne or purple striae were found. Serum levels of cortisol, aldosterone, angiotensin I/II, renin activity and catecholamine were normal. Urinary levels of vanillylmandelic acid (VMA), catecholamines and other metabolites were normal except for slightly elevated 17-ketocorticosteroid and 17-hydroxycorticosteroid level (Table I). These results suggested low probability of functional tumors.

The plain CT scan discovered a large well-circumscribed mass $(8.6 \times 7.5 \times 6.5 \mathrm{~cm})$ in the left adrenal gland. The mass had a mixed heterogeneous signal intensity areas with multiple fat signal intensity areas. Postcontrast CT scan found enhancement of the solid portion of the mass (Figure 1a). The left kidney was compressed by the mass and shifted down (Figure 1b).

Phenoxybenzamine (10 $\mathrm{mg} /$ day) was given orally for three days as a precaution for volume expansion, because pheochromocytoma could not be excluded in preoperative evaluation. The mass was completely removed via retroperitoneal laparoscopic approach. The upper pole of left adrenal gland was also excised due to adhesion to the mass. No major vessels were encountered in the operative field, so massive hemorrhage was avoided. The estimated blood loss was $30 \mathrm{ml}$. The operation time was 100 minutes. 
The pathological diagnosis was adrenal myeololipoma with adrenocortical adenoma. Gross specimen consisted of a $10.9 \times 7.2 \times 5.2 \mathrm{~cm}$, grayish yellow and irregular shaped mass with solid texture, and part of left adrenal gland measuring $6.5 \times 4 \times 0.1 \mathrm{~cm}$. Light microscopic findings (Figure 2) revealed the tumour cells arranged in trabecular, nested and organoid patterns. The tumour cells were relatively homogenous, and the intercellular boundaries were unclear. The cytoplasm was eosinophilic and the nucleus was oval-shaped. There was no cellular atypia. Multiple foci of adipose tissue were seen scattered within the tumour, where hematopoietic cells including erythrocytes, leukocytes and platelets were noted in the adipose tissues. Immunostaining revealed tumour cells staining positive for Ki-67 $(+,<1 \%$, hematopoietic tissue: $79 \%$ ), cytokeration (CK), Synaptophysin, Inhibin-a and MelanA, and were negative for $\mathrm{CgA}$, ACTH and S-100 protein.

Table I: Serum cortisol rhythm, supine and orthostatic hypertension test, serum catecholamine concentration, urinary catecholamine and other metabolites concentrations.

\begin{tabular}{|c|c|c|c|c|}
\hline Serum cortisol rhythm & $0: 00$ & $8: 00$ & $16: 00$ & $20: 00$ \\
\hline Serum cortisol concentration ( $\mu \mathrm{g} / \mathrm{dl})$ & 3.07 & 5.37 & 5.50 & 4.17 \\
\hline Supine and orthostatic hypertension test & Standing position & & Lying position & \\
\hline Aldosterone/renin activity & 30.79 & & 54.08 & \\
\hline Aldosterone (ng/dl) & 7.39 & & 7.03 & \\
\hline Angiotensin $137^{\circ} \mathrm{C}(\mathrm{ng} / \mathrm{ml})$ & 2.81 & & 1.92 & \\
\hline Angiotensin II (pg/ml) & 46.19 & & 29.61 & \\
\hline Renin activity (ng/ml/h) & 0.24 & & 0.13 & \\
\hline Angiotensin $14^{\circ} \mathrm{C}(\mathrm{ng} / \mathrm{ml})$ & 2.57 & & 1.79 & \\
\hline Serum catecholamine concentration & day1 & & normal range & \\
\hline Adrenaline $(\mathrm{pg} / \mathrm{ml})$ & 26.51 & & $0.00-100.00$ & \\
\hline Norepinephrine (pg/ml) & 101.34 & & $0.00-600.00$ & \\
\hline Dopamine (pg/ml) & 49.88 & & $0.00-100.00$ & \\
\hline Urinary catecholamine and other metabolites concentration & day1 & day2 & day3 & normal range \\
\hline Vanillylmandelic acid (VMA) (mg/24h urine) & 8.75 & 9.75 & 8.31 & $0.00-13.60$ \\
\hline 17-ketocorticosteroid (17-KS) (mg/24h urine) & $15.87(\uparrow)$ & $22.48(\uparrow)$ & l & (Female $6.00-14.00$ Male $10.00-25.00$ ) \\
\hline 17-hydroxycorticosteroid (17-OHCS) (mg/24h urine) & $10.08(\uparrow)$ & $13.62(\uparrow)$ & I & (Female $2.00-8.00$ Male $3.00-10.00$ ) \\
\hline Adrenaline ( $\mu \mathrm{g} /$ day) & 4.39 & 1 & I & $0.00-20.00$ \\
\hline Norepinephrine ( $\mu \mathrm{g} /$ day) & 65.89 & I & l & $0.00-90.00$ \\
\hline Dopamine ( $\mu \mathrm{g} /$ day) & 410.21 & 1 & 1 & $0.00-600.00$ \\
\hline
\end{tabular}

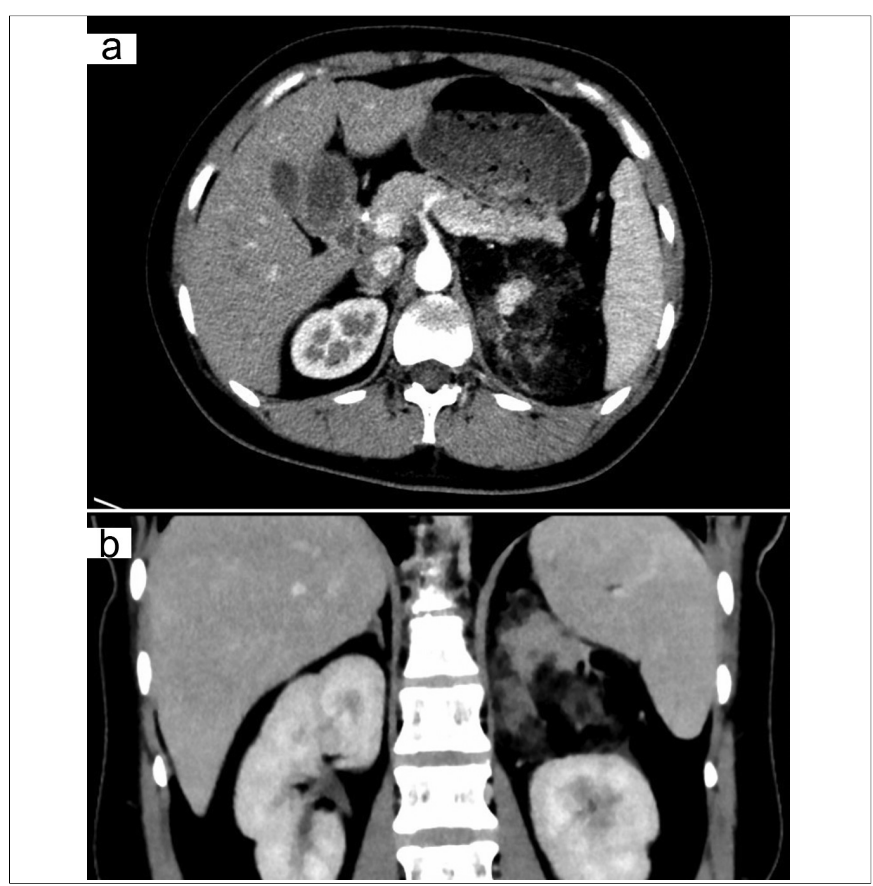

Figure 1: (a) Axial image from post-contrast abdominal CT scan showing enhancement of the solid portion in left adrenal gland, but no enhancement of the fat signal intensity areas. (b) Coronal image from post-contrast abdominal CT scan showing a $8.6 \times 7.5 \times 6.5 \mathrm{~cm}$ mixed heterogeneous signal intensity left adrenal mass with multiple fat signal intensity areas. The left kidney was compressed downwards by the left adrenal gland mass.

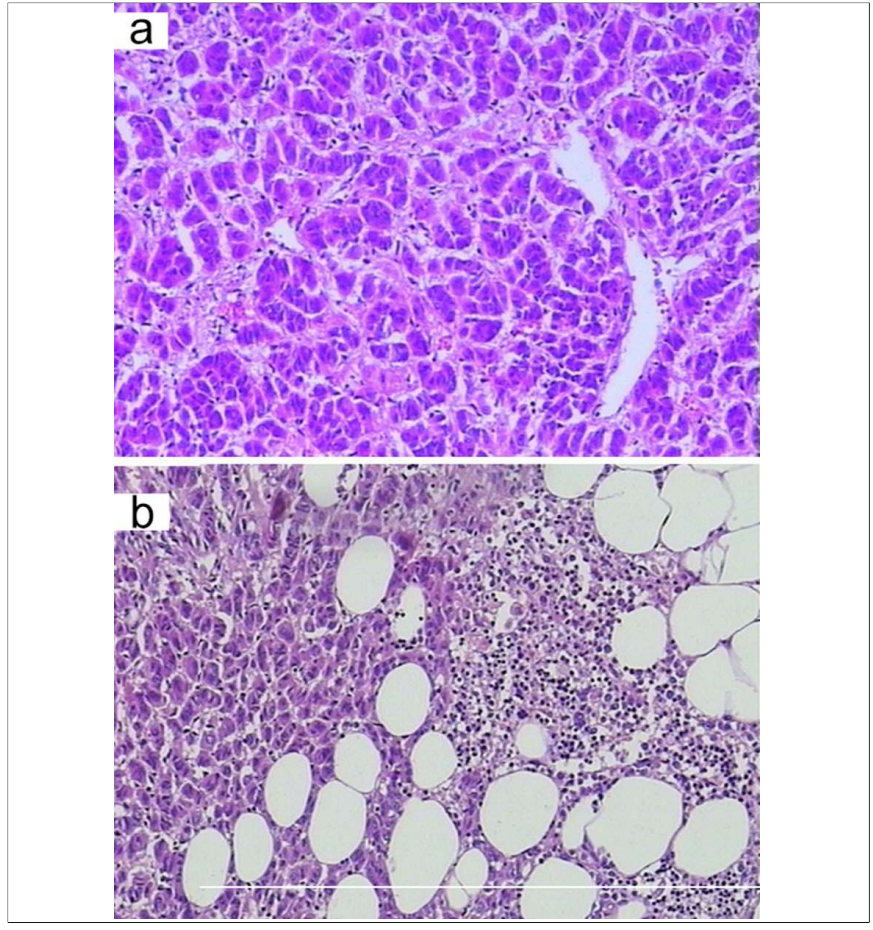

Figure 2: (a) The tumour cells are relatively homogenous in size, and the intercellular borders are unclear. The cytoplasm is eosinophilic and the nucleus oval-shaped. (b) Multiple foci of adipose tissue are seen scattered within the tumour, and containing admixed hematoportic elements. 
Postoperative recovery was uneventful and her BP readings were normal. The patient was discharged on the 5th postoperative day. At 15 months follow-up, the patient was normotensive and there was no recurrence of tumour.

\section{DISCUSSION}

Adrenal myelolipoma is a rare benign tumour of adrenal gland, initially described by a German pathologist, Edgar von Gierke, in 1905. The term "myelolipoma" was used by a French pathologist, Charles Oberling, in 1929.5 Adrenal myelolipoma is uncommon, with a few hundred cases been reported in the past.4-8 This makes the synchronous occurrence of adrenal adenoma and adrenal myelolipoma within the same adrenal gland even more rare. Only approximately 20 such cases have been documented, and most have either presented with Cushing's syndrome or Conn's syndrome.2,4,6 In the documented cases, the tumour was often small with diameter ranging from 5 to $30 \mathrm{~mm} \cdot 2,4$ To the best of the authors' knowledge, the largest one documented was $12 \mathrm{~cm}$ in size ${ }^{6}$ and the second largest one was $8.5 \mathrm{~cm} .^{7}$ In this case, the patient presented with hypertension only, with no other evidence of functional adrenal cortical adenoma, and the greatest diameter of the tumour was $8.6 \mathrm{~cm}$. These make this case one another unique addition to the previous reported cases.

With the increased usage and sensitivity of CT and MRI, adrenal incidentalomas are increasingly being picked up. Adrenal myelolipoma together with adrenal adenoma as collision tumour is noted to occur more common in females, and in the left adrenal gland. ${ }^{4}$ This case is consistent with these characteristics.

Surgery may be necessary, if the tumour is symptomatic or larger than $7 \mathrm{~cm}$ due to increased risk of spontaneous rupture with retroperitoneal hemorrhage. ${ }^{9}$ In the study hospital, the authors usually operate on patients whose tumours are larger than $5 \mathrm{~cm}$. In this case, the size of the left adrenal mass was $8.6 \times 7.5 \times 6.5 \mathrm{~cm}$ in preoperative $\mathrm{CT}$, and the patient was stage 2 hypertensive which could be secondary to external renal compression by the adrenal mass. ${ }^{10}$ Therefore, surgery was performed.

According to the latest World Health Organization classification of tumours of endocrine organs, adrenal myelolipoma is the most common type among different adrenal lipomatous tumours. It is composed of a mixture of adipose tissue and hematopoietic elements. ${ }^{2}$ In this case, the tumour had pathological characteristics of both adrenal myelolipoma and adrenocortical adenoma. It was composed of well-differentiated adenoma cells, adipose tissue and hematopoietic elements.

In conclusion, the rare association of adreno-cortical adenoma and adrenal myelolipoma should be emphasised, and concurrence of these two types of tumours is often discovered incidentally with CT or MRI. It is more common in females, and in left adrenal gland. Though usually benign, the duo may present with endocrine dysfunction, such as Cushing's syndrome. Therefore, consulting endocrinologists for endocrine dysfunction is helpful to improve the overall outcome. Surgical resection is reserved for symptomatic tumours or large myelolipoma (>7 cm in size). The final diagnosis mainly relies on pathological examination.

\section{REFERENCES}

1. Abecassis $M$, McLoughlin MJ, Langer B, Kudlow JE. Serendipitous adrenal masses: Prevalence, significance, and management. Am J Surg 1985; 149:783-8.

2. Lam AK. Lipomatous tumours in adrenal gland: WHO updates and clinical implications. Endocr Relat Cancer 2017; 24:R65R79.

3. Wani NA, Kosar T, Rawa IA, Qayum A. Giant adrenal myelolipoma: Incidentaloma with a rare incidental association. Urol Ann 2010; 2:130-3.

4. Lu HS, Gan MF, Chen HS, Huang SQ. Adrenal myelolipoma within myxoid cortical adenoma associated with Conn's syndrome. J Zhejiang Univ Sci B 2008; 9:500-5.

5. Kenney PJ, Wagner BJ, Rao P, Heffess CS. Myelolipoma: CT and pathologic features. Radiology 1998; 208:87-95.

6. Ong K, Tan KB, Putti TC. Myelolipoma within a non-functional adrenal cortical adenoma. Singapore Med J 2007; 48:e200-2.

7. Manassero F, Pomara G, Rappa F, Cuttano MG, Crisci A, Selli C. Adrenal myelolipoma associated with adenoma. Int $J$ Urol 2004; 11:326-8.

8. Settakorn J, Sirivanichai C, Rangdaeng S, Chaiwun B. Fineneedle aspiration cytology of adrenal myelolipoma: Case report and review of the literature. Diagn Cytopathol 1999; 21: 409-12.

9. Daneshmand S, Quek ML. Adrenal myelolipoma: Diagnosis and management. Urol J 2006; 3:71-4.

10. Patel TV, Goes N. Page kidney. Kidney Int 2007; 72:1562. 